\title{
Auscultating heart and breath sounds through patients' gowns: who does this and does it matter?
}

Authors: Alastair J Rankin ${ }^{\ddagger 1}$, Stephen H Rankin², Andrew C Rankin ${ }^{3}$

1) Department of Medicine, Western Infirmary, Glasgow, UK, G 11 6NT

2) Department of Medicine, Glasgow Royal Infirmary, Glasgow, UK, G 4 OSF

3) School of Medicine, University of G lasgow, UK, G $128 Q \mathrm{Q}$

${ }^{\ddagger}$ Author for correspondence:

Email: alastair.rankin@ nhs.net Tel: +44-141-211-4833

Keywords: physical examination, auscultation, heart sounds, heart murmur Word count: 2143

Tables: 1

Figures: 3 
Main messages

- A majority of doctors of all grades auscultate patients' heart and breath sounds through a hospital gown, at least sometimes.

- The main reasons for this practice are compassionate rather than expediency

- The majority of doctors could not differentiate between skin or gown recordings

- The quality of auscultation is adversely affected by listening through clothing

Current research questions

- Would cardiac auscultation via a gown affect the diagnosis of murmurs and added sounds in patients with valvular heart disease? 


\section{Abstract}

\section{Background}

Doctors are taught to auscultate with the stethoscope applied to the skin, but in practice may be seen applying the stethoscope to gown.

\section{Objectives}

To determine how often doctors auscultate heart and breath sounds through patients' gowns and to assess the impact of this approach on the quality of the sounds heard.

\section{Methods}

A sample of doctors in the West of Scotland were sent an email in 2014 inviting them to answer an anonymous questionnaire about how they auscultated heart and breath sounds.

Normal heart sounds from 2 subjects were recorded through skin, through skin and gown, and through skin, gown and dressing gown. These were played to doctors, unaware of the origin of each recording, who completed a questionnaire about the method and quality of the sounds they heard.

\section{Results}

206 of $445(46 \%)$ doctors completed the questionnaire. $124(60 \%)$, stated that they listened to a patients' heart sounds, and 156 (76\%) to breath sounds, through patients' gowns. Trainees were more likely to do this compared to consultants (OR 3.39, 95\% CI 1.74-6.65). Doctors of all grades considered this practice affected the quality of the sounds heard.

32 doctors listened to the recorded heart sounds. 23 of the 64 (36\%) skin and 23 of the $64(36 \%)$ gown recordings were identified. The majority of doctors $(74 \%)$ could not differentiate between skin or gown recordings, but could tell them apart from the double layer recordings $(p=0.02)$. Trainees were more likely to hear artefactual added sounds $(p=0.04)$.

\section{Conclusions}


Many doctors listen to patients' heart and breath sounds through hospital gowns, at least occasionally. In a short test, most doctors could not distinguish between sounds heard through a gown or skin. Further work is needed to determine the impact of this approach to auscultation on the identification of murmurs and added sounds. 


\section{INTRODUCTION}

Standard clinical skills teaching is that cardiac auscultation should be performed with the stethoscope applied directly to the skin of the patient, with the chest fully exposed [1]. However, doctors of all grades have been observed auscultating the heart and chest of patients through a hospital gown [2]. It is not known how common the practice of auscultation through a gown is, what the reasons for the practice are, and what effect it has on the clinical value of auscultation.

The invention of the stethoscope by Laennec in 1816 was prompted by his desire to avoid the embarrassment that may have been caused by direct auscultation, with the ear to the chest, when examining a young female patient $[3,4]$. A similar motive may underlie the reluctance to expose the patient by removal of all clothing from the upper chest. There are the further advantages of speed of clinical examination and the minimisation of disruption to the patient. An additional factor may be the decline in confidence in clinical examination in the era of advancing technology, improved imaging [5] and "gadgetophilia" [6]. However there are concerns about the practice as there may be loss of acoustic quality, the inability to utilise the variation of pressure applied to the skin to vary the frequency response and the creation of background noise and added sounds.

Listening with a stethoscope through the hospital gown contradicts traditional teaching. Chizner offers balanced advice stating: "The patient should be properly gowned to avoid embarrassment... proper auscultation should not be done through clothing. For maximal auscultatory yield, the stethoscope should be touching the patient's bare chest" [7]. However, there are clear advantages of listening through the gown, including those of efficiency and patient privacy. The General Medical Council's guidance "Good Medical Practice" advises judgment should be used to balance the need for a "good standard of practice", with the requirement to respect "the dignity and privacy" of your patient [8]. 
The aim of this study was to determine how often doctors auscultate through patients' gowns and to assess the impact of this approach on the quality of the heard sounds heard.

\section{METHODS}

Participants. Doctors of all grades whose email addresses were available to the investigators from local hospital rota lists in the West of Scotland were invited by email to complete an anonymous online questionnaire in September 2014. The majority of those doctors worked in two Glasgow hospitals (see box). A smaller number of doctors in these 2 hospitals were invited in person by the researchers to listen to recorded heart sounds and to complete a questionnaire about the recordings.

Questionnaire. An on-line questionnaire was created via the website www.surveymonkey.com (Figure 1). Participants were asked their grade, whether they listened to a patient's heart or breath sounds via the patient's hospital gown and, if so, how often. They were asked their main reason for doing this, and whether they thought it affected the quality of auscultation. All questions required an answer to complete the survey. The final section invited optional free-text comments. Non-responders were not followed up.

Electronic recordings of heart sounds. Recordings were made using a Littmann electronic stethoscope, model 3200, of heart sounds from 2 male subjects, aged 27 and 62 years, with structurally normal hearts at echocardiography. The mitral area (left $5^{\text {th }}$ intercostal space in the midclavicular line) was used for all recordings. Recordings of 30 seconds duration were made with the stethoscope placed over each of 3 surfaces: skin, hospital gown, hospital gown plus a dressing gown. The software supplied with the stethoscope, Zargis StethAssist Software Version 1.0 (Zargis Medical group, P rinceton, USA), was used to store, analyse and play back the resulting files. It also allowed recordings to be viewed in spectographic mode for visual assessment. 


\section{Differentiating heart sounds heard through skin with or without gowns.}

The six recordings (via skin, hospital gown, and hospital gown plus dressing gown for the 2 subjects) were played back to volunteers using the electronic stethoscope. The order of playback for each subject was randomized, with each participant listening to the same order of recordings (subject 1 followed by subject 2). Participants were asked to state which surface was used for each recording. They were asked if each recording was of diagnostic quality, and if they heard any added sounds such as systolic or diastolic murmurs, pericardial rub or $3^{\text {rd }}$ heart sounds.

\section{Data analysis}

The results of the completed on-line questionnaires were downloaded from the SurveyMonkey website for off-line analysis. The results of the recording playback study were obtained from the questionnaires completed by the participants. Spreadsheets and figures were created using Microsoft Excel 2011 Software (Microsoft, USA).

\section{Statistical Analysis}

Descriptive statistics and odds ratios (OR) were produced, where appropriate. Reported OR and the associated $95 \%$ confidence intervals ( $95 \% \mathrm{Cls}$ ) express the odds of respondents giving a specified answer based on their grade. The ORs were calculated using binary logistic regression. Pearson chi-squared analysis was used for non-parametric nominal data. Analyses were undertaken using IBM SPSS (version 22, New York).

\section{Ethical permission}

Ethical approval was obtained from the Medical, Veterinary and Life Sciences College Ethics Committee, University of Glasgow. Informed consent was obtained.

\section{RESULTS}

Questionnaire 
Participants. 445 doctors were invited to take part and 206 (46\%) completed the questionnaire. $58(28 \%)$ were foundation year (FY) doctors; 54 (26\%) were core medical trainees (CMT) or general practitioner specialty trainees (GPST); 46 (22\%) were specialist trainees (ST); and 48 (23\%) were consultants.

\section{Auscultation of heart and breath sounds through patients' gowns. 124} $(60 \%)$ stated that they listened to a patient's heart sounds and $156(76 \%)$ that they listened to breath sounds through the patient's hospital gown, at least 'sometimes' (Figure 2). 48 (23\%) 'often' listen through a patient's hospital gown (at least 1 in 10 patients) or more frequently; 1 respondent listened through the gown 'most of the time'. Only 39 (19\%) respondents stated that they never listened to either heart or breath sounds through a gown, 19 of which were consultants. Trainees were 3 times more likely to auscultate heart sounds through a hospital gown than consultants (OR 3.39, 95\% CI 1.746.65), and almost 4 times more likely to listen to breath sounds through the gown than consultants (OR 3.93; 95\% Cl 2.0-7.9).

Reasons for listening through gown. The main reasons given by 131 (64\%) participants for this practice were compassionate: patient privacy (38), patient immobility (70) and inappropriate setting for examination (23). 31 (15\%) stated their main reason was to save time. 'Other' was selected by $44(21 \%)$, of whom 30 commented that they never did it, and 7 indicated they did it for multiple reasons.

\section{Perceived effect of listening through a gown on quality. 187 (91\%)} participants thought that listening through the hospital gown affected the quality of the sounds heard (Figure 2). 52 (25\%) thought that doing so affected the quality substantially ("Yes, a lot"), of which 27 (52\%) admitted to the practice. Responders were invited to leave comments and 37 (18\%) did so (Table 1 ).

\section{Table 1 - selection of comments}


o never!

o admit doing this very occasionally... / think the sounds are muffled and liable to extraneous rustling

- Gowns are so thin that quality of sound is not affected when using a high quality stethoscope

o It is a bit like wearing gloves for taking bloods. It initially seems to make things more difficult but as the years have gone on I find I am not missing things compared to colleagues/Echo reports.

o Should I not hear clearly... I would proceed to listen under the hospital gown

- Male doctor examining a female patient, chaperone not always available therefore at times it is not possible to fully expose patient.

- I would fail a candidate outright in finals or MRCP PACES for listening through clothes. No exceptions. Lazy and sloppy

o The only people / see doing this are lazy consultants on a post take WR!

o I hear creps every time I listen through a piece of clothing, so don't anymore

o I have been criticised for listening through clothing, but this was after having witnessed the same repeatedly by all grades of doctor in hospital.

o Never do it. I've tested it years ago. Appalling practice to listen through clothes.

\section{Differentiating heart sounds heard through skin with or without gowns.}

Spectrographic display of recordings. Qualitative visual analysis of the recorded heart sounds showed first and second heart sounds were clearly identified in each of the recordings, and confirmed the absence of murmurs (Figure 3). With auscultation via gown, there were visible increases in both low and high amplitude sounds, particularly with the recording through the double layers.

\section{Identification of skin, gown or double layer recordings by doctors. 32} doctors listened to the recorded heart sounds. There were $7 \mathrm{FY}$ doctors, 8 GPST/CMT, 7 ST and 10 consultants. Each doctor listened to 3 recordings (skin, gown, gown plus dressing gown) from 2 subjects. One doctor (FY1) identified all recordings correctly. Only 23 of the $64(36 \%)$ recordings through 
skin, and 23 of the $64(36 \%)$ recordings through skin and gown, were identified correctly. 34 of the $64(53 \%)$ recordings through the double layers were identified. When the recordings through skin were considered together with the recordings through skin plus gown, 95 of the 128 (74\%) were identified as being either through skin or through gown, and not through the double layer. The majority could not differentiate between skin and gown, but could tell them apart from the double layer recordings $\left(C^{2}=5.186\right.$; $d f=1, p=0.02$ ). This is reflected in the assessment of the quality of the heart sounds heard in the recordings. They were considered to be of diagnostic quality in 114 (59\%) of recordings; $64 \%$ of skin, $70 \%$ of gown and $44 \%$ of double layer.

Grade of doctor and interpretation of recorded heart sounds. Correct identification of recordings of heart sounds through skin was made in 25 (60\%) recordings by FY, in 19 (40\%) by GPST/CMT, in 11 (26\%) by ST doctors and in $22(37 \%)$ by consultants. There was no difference between trainees and consultants for the correct identification of skin (OR 2.07, 95\% 0.64-6.7). Despite the recordings being of normal heart sounds, added sounds were reported in 75 (39\%) recordings. The most common were systolic murmur $(25 \%)$ and $3^{\text {rd }}$ heart sound $(10 \%)$. Trainees were significantly more likely to hear artefactual sounds than consultants $(p=0.04)$. The incidence of added sounds across all recordings increased from 21 for skin, to 26 for gown and 28 for double layer recordings. Trainees were 3 times more likely to hear artefactual added sounds than consultants when listening through both layers (OR 3.29, 95\% CI 1.02-10.6; $\left(C^{2}=4.156, d f=1, p=0.04\right)$ ), but there was no difference for skin (OR $1.71,95 \% \mathrm{Cl} 0.52-5.60)$ or gown (OR $1.34,95 \% \mathrm{Cl} 0.46-3.86)$.

\section{DISCUSSION}

This study reports that the majority of doctors auscultate patients, heart and breath sounds through hospital gowns, at least "sometimes", with nearly a quarter doing it "often". Consultants are less likely to do this than trainees. The main reasons for doing so were compassionate, with patient immobility 
being most common. Despite the prevalence of this practice, 9 out of 10 doctors think that it does affect the quality of auscultation. Contrary to this belief, doctors of all grades were unable to differentiate between heart sounds recorded via skin compared to through a hospital gown. Doctors were better at differentiating recordings taken via 'double layers' (hospital gown and dressing gown), which was associated with a lower diagnostic quality, and with an increase in the number of added sounds.

Doctors are more likely to listen to breath sounds than heart sounds through patients' gowns. Listening to heart sounds through a gown carries the risk of missing soft added sounds; listening to breath sounds adds the risk of mistaking sounds caused by the rubbing of gown on stethoscope as pulmonary crackles. Stethoscope technology has advanced $[9,10]$ and the higher acuity instruments may be able to compensate for a reduction in surface quality. However, in the real-life working environment, with background noise, it is desirable to optimise auscultatory quality, and therefore listen via skin.

The limitations of the questionnaire study include the risk of self-selection bias, resulting in responses from clinicians with active interest, and socialdesirability bias, with responders answering in a way that they feel they should rather than what they actually do. The survey was anonymised to try to minimise this bias. The playback phase of the study also has limitations. There is evidence supporting the use of electronic stethoscopes [11], but the playback of recorded heart sounds may not recreate the authentic experience of live auscultation, as indicated by the number (36\%) who felt that the skin recordings were not of diagnostic quality. The participants were observed by an investigator during the playback study which may have had an effect on their performance ('the Hawthorne effect' [12]). This may have contributed to the junior doctors reporting added sounds more frequently. The use of normal heart sounds for playback means that we did not assess how the accuracy of detection of abnormal heart sounds would vary with skin or gown recording, nor the impact on diagnosis and clinical management. A previous study using recordings via skin of cardiac murmurs and extra sounds showed that the 
auscultatory proficiency of trainee doctors was poor, with UK trainees correctly identifying only $20 \%$ of recordings [13]. The present study focused on the effects on auscultatory quality to avoid the confounder of diagnostic skills; the impact of listening through a gown on diagnosis and management is an area of future research.

There has been a gradual decline in auscultatory skills [14]. In patients with confirmed mitral regurgitation at echocardiography, only one third of doctors heard a murmur [15]. The increasing use of imaging, in particular echocardiography, may mean doctors rely less on their clinical findings [5]. Good clinical examination still has a role in patient care. It increases the pretest probability of investigations, reduces the cost burden to health care providers and remains important in settings without immediate access to imaging, such as the initial assessment of hospitalized patients and doctors in primary care.

The gradual decline in auscultatory skills may be explained by the increasing use of imaging, in particular echocardiography. [14]. In patients with confirmed mitral regurgitation at echocardiography, only one third of doctors heard a murmur [15]. Good clinical examination still has a role in patient care. It increases the pre-test probability of investigations, reduces the cost burden to health care providers and remains important in settings without immediate access to imaging, such as the initial assessment of hospitalized patients and doctors in primary care. 


\section{Competing interests}

None.

\section{Funding}

None.

\section{Contributorship}

All authors contributed to the planning, executing, analysing and writing of this study.

\section{Acknowledgements}

The authors wish to thank all those who participated in the survey. A special thanks to Dr Ross Campbell for assistance with echocardiography, and $\mathrm{Mr}$ David Rankin for providing the Bluetooth dongle.

\section{Context of this study:}

Glasgow R oyal Infirmary and the Western Infirmary, Glasgow, are large urban teaching hospitals located in central Glasgow, Scotland, United Kingdom. 
Figure 1.

This anonymous on-line questionnaire was used to determine the frequency of the practice of auscultation through a hospital gown, the main reasons for this practice and whether doctors felt it affected auscultatory quality. 


\section{Figure 2.}

Bar chart showing the incidence of auscultation, at least "sometimes", through hospital gown for heart sounds (red) and breath sounds (blue) by doctors' training grade and the percentage of each grade that felt it affected quality. $[\mathrm{FY}=$ foundation year trainee; $\mathrm{CMT}=$ core medical trainee; GPST = general practice specialty trainee; $\mathrm{ST} 3+=$ specialist trainee year 3 and above]. 


\section{Figure 3. Visual Analysis of recordings}

Recordings taken from skin (top panel), gown (middle panel) and double layer (bottom panel) are shown in spectographic mode. The first 5 seconds of the recording were removed to eliminate added noise due to stethoscope placement. The first half of each panel displays an increase in both background noise and added sounds with the recordings via additional layers. The second half of each panel is annotated to show $1^{\text {st }}$ and $2^{\text {nd }}$ heart sounds (white arrows). The '?' symbol is above artefactual added sounds which may confuse the listener into hearing murmurs or added sounds that are not cardiac in origin. 


\section{References}

1. Perloff J K, Braunwald E. Physical examination of the heart and circulation. In: Braunwald $E$, ed. Heart Disease, A textbook of cardiovascular medicine ( $5^{\text {th }}$ edition). Philadelphia; WB Saunders 1997: 15-52.

2. Grais IM. Proper use of the stethoscope; three heads and one tale. Texas Heart Institute J ournal 2013;40:120-2.

3. Cheng TO. How Laennec invented the stethoscope. International J Cardiol 2007;118:281-5.

4. Welsby PD, Parry G, S mith D. The stethoscope: some preliminary investigations. Postgrad Med J 2003;79:695-8.

5. Feddock CA. The lost art of clinical skills. Amer J Med 2007;120:374-8.

6. Cheng TO. Seeing is not believing, but hearing is: The stethoscope is still useful. International J Cardiol 2013;165:1-2.

7. Chizner MA. Cardiac auscultation: rediscovering the lost art. Curr Probl Cardiol 2008;33:326-408.

8. General Medical Council (2013) Good Medical Practice. London, GMC

9. Spencer WH. On a New Form of Stethoscope in its Relations to the Theory and Practice of Auscultation. BMJ 1874;691:409-411.

10. A bella M, Formolo J , Penney DG. Comparison of the acoustic properties of six popular stethoscopes. I Acoust Soc Am 1992;91:2224-8.

11. Iversen K, Greibe R, Timm HB, Skovgaard LT, Dalsgaard M, Hendriksen $\mathrm{KV}$ et al. A randomized trial comparing electronic and conventional stethoscopes. Am J med 2005;118:1289

12. Sedgwick P. The Hawthorne effect. BMJ 2012;344:d8262

13. Mangione S. Cardiac Auscultatory S kills of P hysicians-in-Training: A Comparison of Three English-Speaking Countries. Am J Med. 2001;110:210-216.

14. DeMaria AN. Wither the cardiac physical examination? J Am Coll Cardiol 2006;48:2156-2157

15. Codispoti CA, Eckart RE, Rutberg SA, Shry EA, Boyd SYN. Appreciation of precordia cardiac murmur on examination relative to knowledge of valvular heart disease. Cardiology in Review 2005;13:147-151. 
Figure 1. Survey Questionnaire

1. What grade are you?

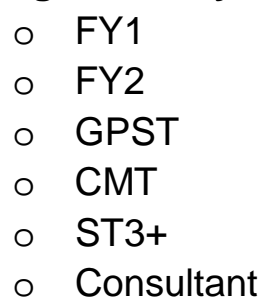

- Specialty (where applicable):

2. When listening to a patient's heart sounds do you ever place the stethoscope over the patient's hospital gown? (i.e. do you listen through the gown? )

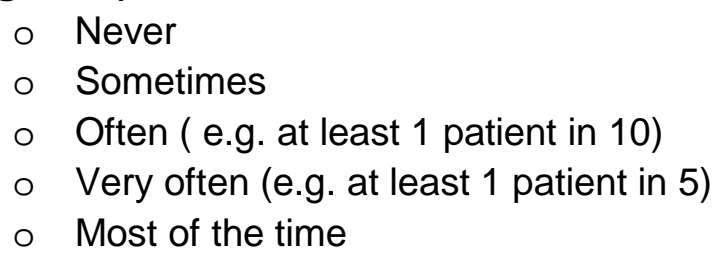

3. When listening to a patient's breath sounds do you ever place the stethoscope over the patient's hospital gown? (i.e. do you listen through the gown? )

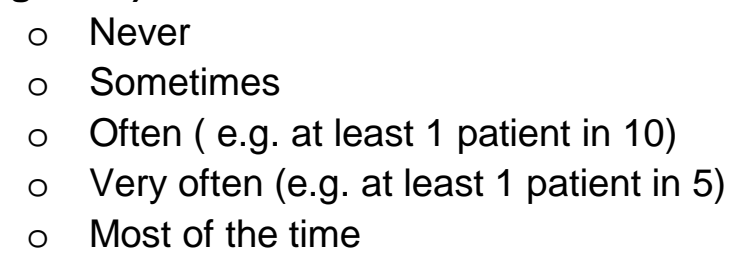

4. If you ever listen through a patient's hospital gown what is the main reason for doing this?
o To save time
o Patient privacy
o Inappropriate setting for examination
o Patient immobility
o Other (please specify)

5. Do you think it affects the quality of auscultation if you place the stethoscope on top of the hospital gown?
o No
o Yes, a little
o Yes, a lot
o Not sure

6. The investigators welcome any comments you may have. Please feel free to tell us why you selected any of the answers that you did. 